\title{
VHE gamma-ray measurements of the Crab Nebula and Pulsar by MAGIC
}

\author{
G. Giavitto*, S. Klepser*,†, M. Lopez**, D. Mazin", ${ }^{*}$, T. Saito ${ }^{\ddagger}$, \\ T. Schweizer ${ }^{\ddagger}$, R. Zanin ${ }^{\S}$, K. Hirotani $i^{\mathbb{I l}}$, D. Horns" ${ }^{\|}$, M. Meyer ${ }^{\|}$and \\ J. Martín Rodríguez \\ *IFAE, Edifici Cn., Campus UAB, E-08193 Bellaterra, Spain \\ ${ }^{\dagger}$ Deutsches Elektronen-Synchrotron (DESY), D-15738 Zeuthen, Germany \\ **Universidad Complutense, E-28040 Madrid, Spain \\ ${ }^{\ddagger}$ Max-Planck-Institut für Physik, D-80805 München, Germany \\ ${ }^{\S}$ Universitat de Barcelona (ICC/IEEC), E-08028 Barcelona, Spain \\ ${ }^{\llbracket}$ ASIAA/National Tsing Hua University-TIARA, P.O. Box 23-141, Taipei, Taiwan \\ Universtät Hamburg, D-20148 Hamburg, Germany \\ ${ }^{\dagger}$ IEEC/ICE, Edifici Cn., Campus UAB, E-08193 Bellaterra, Spain
}

\begin{abstract}
In the last two years 2010 and 2011 the Crab nebula and its pulsar have surprised the gamma-ray astrophysics community at least twice: the former with $\mathrm{GeV}$ flares detected by AGILE and Fermi-LAT, the latter with very high energy (VHE, E $>100 \mathrm{GeV}$ ) pulsed emission extending up to $400 \mathrm{GeV}$ detected by MAGIC and VERITAS. These exciting results show that there is still much left to understand about this object. We report here the results of the MAGIC stereoscopic observations of the Crab nebula and pulsar carried on between October 2009 and April 2011. For the nebula, the analysis of these data yields a differential energy spectrum with an unprecedented precision spanning from $50 \mathrm{GeV}$ to $45 \mathrm{TeV}$, a precise determination of its inverse Compton (IC) peak, and the measurement of the VHE emission during the GeV flare of April 2011. Implications on the modeling of the Crab nebula, especially regarding the strength of the magnetic field, are discussed. For the pulsar, a phase-resolved energy spectrum and a lightcurve in the range 50-400 $\mathrm{GeV}$ are obtained. Together with previous MAGIC monoscopic results above $25 \mathrm{GeV}$ they allow for a detailed study of the pulsed VHE emission, which can be interpreted as an additional component due to IC emission from secondary and tertiary pairs on IR-UV photons in the outer magnetosphere.
\end{abstract}

Keywords: Gamma-rays,PWN,pulsars,VHE,Crab Nebula,M1

PACS: 95.55.Ka,95.85.-e,95.85.Pw,95.30.Gv,95.30.Qd,04.40.Dg

\section{MAGIC OBSERVATIONS OF THE CRAB NEBULA}

The Crab pulsar and its pulsar wind nebula (PWN) are the remnants of the historical supernova SN 1054[1]. Due to its apparent steady emission, the nebula has been used as "standard candle" for ground-based VHE astronomy since its discovery [2], and it is arguably one of the best studied pulsar - PWN complex in across the whole accessible electromagnetic spectrum.

The MAGIC telescopes situated on the island of La Palma $\left(28.8^{\circ} \mathrm{N}, 17.8^{\circ} \mathrm{W}, 2220\right.$ $\mathrm{m}$ a.s.1.) use the imaging atmospheric Cherenkov technique to detect gamma rays above a few tens of $\mathrm{GeV}$. The two $17 \mathrm{~m}$ telescopes operate in stereoscopic mode, i.e. in coin- 
cidence, achieving a sensitivity ${ }^{1}$ of $0.8 \%$ Crab nebula units above $250 \mathrm{GeV}$ and a lower energy threshold of $50 \mathrm{GeV}$ [3].

We considered 48.7 hours of optimal quality wobble data at zenith angles between $5^{\circ}$ and $50^{\circ}$. The analysis of the data was performed by using the standard tools of the MAGIC analysis software MARS [4]. For the image cleaning a new algorithm (dubbed "sum cleaning") was employed to improve the analysis sensitivity at energies below 100 $\mathrm{GeV}[5,6]$.

The spectrum we obtained spans three decades in energy, from $50 \mathrm{GeV}$ to $45 \mathrm{TeV}$, and 8 decades in flux (see figure 1). It can be fitted to a log-parabola function: $\frac{\mathrm{d} N}{\mathrm{~d} E \mathrm{~d} t \mathrm{~d} A}=$ $f_{0}\left(\frac{E}{1 \mathrm{TeV}}\right)^{\alpha+\beta \log _{10}\left(\frac{E}{1 \mathrm{TeV}}\right)} \frac{\text { photons }}{\mathrm{TeV} \mathrm{cm}^{2}}$, with $f_{0}=3.20 \pm 0.5 \times 10^{11}, \alpha=-2.40 \pm 0.01$ and $\beta=-0.13 \pm 0.02$. The errors on the fit parameters are statistical only, and are quite small $(\sim 5 \%)$ below $100 \mathrm{GeV}$. The systematic errors dominate this measurement, affecting the flux normalization $f_{0}$ and the energy scale by about $15 \%$ and the photon index by \pm 0.15 . If we combine this data with that of Fermi-LAT [7], it is possible to map most of the inverse Compton (IC) bump, and measure its peak position to be $59 \pm 6_{\text {stat }} \pm 10_{\text {sys }} \mathrm{GeV}$.

To model gamma-ray emission of the Crab Nebula, two possibilities were taken into consideration, following [8] (figure $1 \mathrm{~B}$ ): a phenomenological constant magnetic field model, as in [11], and a magnetohydrodynamic (MHD) flow model, as outlined in $[12,13]$. The free parameters for these models (average B-field and magnetization of the flow at the shock, $\sigma$ ) were fitted to the data points obtaining $B=124 \pm 6 \mu G$ and $\sigma=0.0045 \pm 0.0003$ (statistical errors only). Taking systematic uncertainties into account, both models can reproduce the observation: they can only be disentangled by more precise measurements of the Crab and of other joung PWN with smaller systematic errors, to be carried on in the future.

During the September 2010 flare above $100 \mathrm{MeV}$ [14, 15] the gamma-ray flux above $300 \mathrm{GeV}$ from the Crab nebula has been found to be constant within a systematic uncertainty of $13 \%$, to a CL of $95 \%$. Additionally, we observed the nebula during the April 2011 flare [16], under strong moonlight conditions, for a total of about 140 minutes. Also in this dataset we found no evidence of change in the flux between 0.7 and $10 \mathrm{TeV}$ during the first peak of the flare, between April 12 and 14.

\section{MAGIC OBSERVATIONS OF THE CRAB PULSAR}

The Crab pulsar, despite numerous efforts, was never detected above $10 \mathrm{GeV}$ until 2008 [17], because its spectrum shows a steepening at these energies. In fact, the origin of the gamma-ray emission in the "classic" Outer Gap (OG) [18] and Slot Gap (SG) [19] models is curvature radiation, which implies an exponential cutoff at a few GeV. However, during the last year, both operating Cherenkov Telescopes in the northern hemisphere, VERITAS and MAGIC, reported the detection of pulsed gamma-ray emission from the Crab Pulsar, from 25 up to $400 \mathrm{GeV}[10,20]$, inconsistent with such a cutoff shape.

We focus here on the MAGIC stereoscopic results only. Of the above-mentioned ob-

\footnotetext{
${ }^{1}$ Defined as the source strength needed to achieve $N e x / \sqrt{N b k g}=5$ in 50 h effective on-time
} 

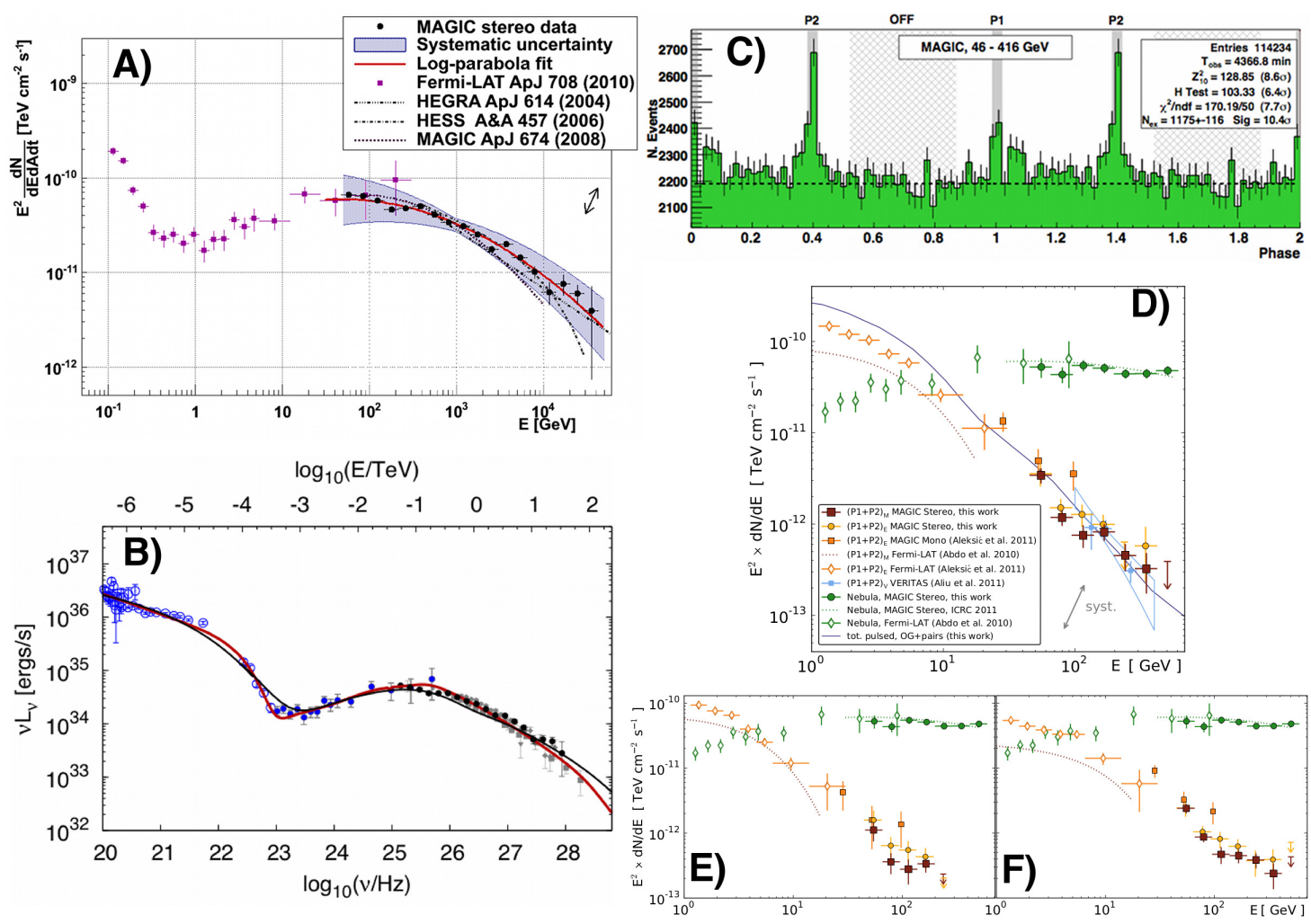

FIGURE 1. A) Spectral energy distribution of the Crab Nebula. B) Models from [8] overlayed to the data in A): constant B-field (red line) and MHD (black line) C) Folded light curve of the Crab pulsar for the total range in estimated energy. D) Compilation of spectral measurements of the Crab pulsar and nebula for both peaks. E) Spectra for P1 only F) Spectra for P2 only. The phase interval definitions are $\mathrm{E}=\mathrm{EGRET}$ [9] M=MAGIC (this work) V=VERITAS[10].

servations, we selected 73 hours of data between both in "on" and "wobble" mode, with zenith angle between $5^{\circ}$ and $35^{\circ}$. The data analysis was performed in the same way as for the nebula, however the cuts used to extract the signal were loosened, and only the energy range $46-416 \mathrm{GeV}$ was considered. The data was timed using the Jodrell Bank ephemeris and the TEMPO2 timing package[21]; it showed a pulsation whose significance was tested with the $\mathrm{Z}_{10}, \mathrm{H}$ and $\chi^{2}$ tests, obtaining 8.6, 6.4 and $7.7 \sigma$, respectively. The folded lightcurve (figure $1 \mathrm{C}$ ) shows two prominent peaks, P1 and P2: they were fitted with two Gaussians, the resulting peak positions in phase were $0.005 \pm 0.003$ and $0.3996 \pm 0.0014$, while the corresponding FWHMs were $0.025 \pm 0.007$ and $0.026 \pm 0.004$.

To extract the spectra we considered two definitions for the phase intervals of the two peaks: one based on the EGRET data [9] (therefore a-priori); another, much narrower, defined a-posteriori as the Gaussian peak positions $\pm 2 \sigma$. The resulting phase-resolved spectra were found to be compatible with power-laws, as can be seen from figure $1 \mathrm{D}$ ), E) and F). More detailed information about this measurement can be found in [20].

Our spectra, together with the Fermi-LAT measurements in [7] comprise the first 
phase-resolved measurements of the gamma-ray emission from the Crab Pulsar between $100 \mathrm{MeV}$ and $400 \mathrm{GeV}$. We put forward a theoretical explanation for this emission, in the framework of the outer-gap model [22], that has the gamma-rays emitted by inverse Compton scattering of secondary and tertiary electron/positron pairs on IR/UV photons. These higher-order magnetospheric cascades were not taken into account in previous pulsar modeling and can produce a power-law gamma-ray spectrum extending at $\mathrm{TeV}$ energies, shown as a blue line in figure $1 \mathrm{D})$. To test this model it will be important in the future to measure the pulsar at those energies.

\section{ACKNOWLEDGMENTS}

We would like to thank the Instituto de Astrofísica de Canarias for the excellent working conditions at the Observatorio del Roque de los Muchachos in La Palma. The support of the German BMBF and MPG, the Italian INFN, the Swiss National Fund SNF, and the Spanish MICINN is gratefully acknowledged. This work was also supported by the CPAN CSD2007-00042 and MultiDark CSD2009-00064 projects of the Spanish Consolider-Ingenio 2010 programme, by grant DO02-353 of the Bulgarian NSF, by grant 127740 of the Academy of Finland, by the DFG Cluster of Excellence "Origin and Structure of the Universe", by the DFG Collaborative Research Centers SFB823/C4 and SFB876/C3, and by the Polish MNiSzW grant 745/N-HESS-MAGIC/2010/0.

\section{REFERENCES}

1. S. Mitton, The Crab Nebula, Scribner, New York, 1978.

2. T. Weekes, et al., ApJ 342, 379-395 (1989).

3. J. Aleksić, et al., Astropart. Phys. 35, 435-448 (2012).

4. A. Moralejo, et al., "MARS, the MAGIC Analysis and Reconstruction Software," in Proceedings of the 31nd International Cosmic Ray Conference, Lodz, Poland, 2009.

5. S. Lombardi, et al., "Advanced stereoscopic gamma-ray shower analysis with the MAGIC telescopes," in [23].

6. R. Zanin, et al., "MAGIC measurement of the Crab Nebula spectrum over three decades in energy," in [23].

7. A. A. Abdo, et al., ApJ 708, 1254 (2010).

8. M. Meyer, D. Horns, and H.-S. Zechlin, A\&A 523, A2 (2010).

9. J. M. Fierro, et al., ApJ 494, 734 (1998).

10. E. Aliu, et al., Science 334, 69-72 (2011).

11. A. M. Hillas, et al., ApJ 503, 744 (1998).

12. C. F. Kennel, and F. V. Coroniti, ApJ 283, 694-709 (1984).

13. A. M. Atoyan, and F. A. Aharonian, MNRAS 278, 525-541 (1996).

14. M. Tavani, et al., Science 331, 736-739 (2011).

15. A. Abdo, et al., Science 331, 739 (2011).

16. R. Buehler, et al., ApJ 749, 26 (2012).

17. E. Aliu, et al., Science 322, 1221-1224 (2008).

18. H. C. Cheng, K. S., and R. M., ApJ 300, 500-539 (1986).

19. J. Arons, ApJ 266, 215-241 (1983).

20. J. Aleksić, et al., $A \& A$ 540, A69 (2012).

21. G. B. Hobbs, R. T. Edwards, and R. N. Manchester, MNRAS 369, 655-672 (2006).

22. J. Aleksić, et al., ApJ 742, 43 (2011).

23. Proceedings of the 32nd International Cosmic Ray Conference, Beijing, China, 2011. 